\title{
Effects of Cooperation on Visible Light Positioning
}

\author{
Osman Erdem, Musa Furkan Keskin, and Sinan Gezici \\ Department of Electrical and Electronics Engineering \\ Bilkent University, 06800, Ankara, Turkey \\ Emails: \{oerdem,keskin,gezici\}@ee.bilkent.edu.tr
}

\begin{abstract}
In this paper, cooperative localization is proposed for visible light systems. The effects of cooperation on the localization accuracy of visible light positioning systems are illustrated based on a Cramér-Rao lower bound expression. The obtained expression is generic for any three-dimensional configuration and covers all possible cooperation scenarios via definitions of connectivity sets. Numerical results are presented to investigate significance of cooperation in various scenarios.

Index Terms- Positioning, visible light, cooperative localization, estimation, received signal strength.
\end{abstract}

\section{INTRODUCTION}

Accurate wireless positioning is crucial for indoor environments in order to facilitate applications such as patient monitoring, inventory tracking, and robotic control [1]-[3]. Although radio frequency (RF) based solutions are commonly employed for wireless indoor positioning [1], [2], [4], [5], light emitting diode (LED) based visible light positioning (VLP) systems have recently emerged as an appealing alternative [6]. Besides localization, visible light systems can also provide illumination and data communication simultaneously. In addition, they do not incur additional installation costs due to the trend of using LEDs for efficient illumination.

In this paper, cooperative positioning is proposed for VLP systems for improving localization accuracy. In the literature, cooperation techniques are intensely studied for RF based positioning systems (see [7] and references therein). The main idea behind cooperation is to utilize measurements among target nodes (which are to be located) in addition to those between target nodes and reference nodes (which have known locations). In this way, localization accuracy can be enhanced based on the relative location information obtained from measurements among target nodes [7]. There exist numerous algorithms in the literature for various cooperation scenarios and some recent works focus on fundamental limits for cooperative positioning systems [8]-[16]. For example, in [9], network experiments are conducted using ultra-wideband (UWB) signals, and comparisons between cooperative and noncooperative positioning systems are performed to reveal the benefits of cooperative positioning under a common setting. The study in [15] presents an overview of cooperative positioning algorithms from the viewpoint of estimation theory and factor graphs. It proposes a distributed cooperative positioning algorithm and compares its performance against other conventional noncooperative and cooperative positioning techniques.

Although cooperation techniques have been considered for RF based positioning systems, there exist no studies in the literature that perform visible light positioning in the presence of cooperation. In this manuscript, we propose a cooperative VLP system and provide a theoretical analysis by deriving a generic Cramér-Rao lower bound (CRLB) expression. The provided expression is valid for any three-dimensional configuration and covers all possible cooperation scenarios via definitions of connectivity sets. Although CRLB expressions are obtained for VLP systems in [17]-[23], all of them are for noncooperative systems and consider the presence of a single VLP receiver. In particular, [17]-[21] focus on distance estimation between an LED transmitter and a VLP receiver while [22] and [23] derive CRLBs for received signal strength (RSS) based position estimation for a single VLP receiver.

In this manuscript, cooperative positioning is proposed for visible light systems, in which there exist multiple LED transmitters with known locations and multiple visible light communication (VLC) units to be located. Each VLC unit is modeled to contain multiple LEDs and multiple photodetectors (PDs) so that it can communicate with both the LED transmitters at known locations and the other VLC units. By defining a connectivity set for each PD in the VLC units, all possible cooperation scenarios are taken into account. A CRLB expression is derived for estimating the locations of the VLC receivers, and based on the proposed expression, the effects of cooperation are quantified. The provided CRLB expression is generic for any configuration and covers the noncooperative scenario as a special case. Numerical results are presented to investigate the significance of cooperation in various conditions. The main contributions in this paper can be summarized as the proposal of a cooperative VLP system for the first time in the literature, and the derivation of a generic CRLB expression for cooperative VLP systems.

The rest of the manuscript is organized as follows: The system model is described and the CRLB expression is derived in Section II. Numerical results are provided in Section III, and concluding remarks are made in Section IV.

\section{System Model And Theoretical Analysis}

The proposed cooperative VLP system consists of $N_{L}$ LED transmitters and $N_{V}$ VLC units, as illustrated in Fig. 1. The location of the $j$ th LED transmitter is denoted by $\mathbf{y}_{j}$ and its orientation vector is given by $\widetilde{\mathbf{n}}_{T, j}$ for $j \in\left\{1, \ldots, N_{L}\right\}$. The locations and the orientations of the LED transmitters are assumed to be known, which is a reasonable assumption for practical systems [22], [24]. In the proposed system, each VLC unit not only gathers signals from the LED transmitters but also communicates with other VLC units in the system for cooperation purposes. To that aim, VLC units are equipped with both LEDs and PDs; namely, there exist $L_{i}$ LEDs and $K_{i}$ PDs at the $i$ th VLC unit for $i \in\left\{1, \ldots, N_{V}\right\}$. The unknown location of the $i$ th VLC unit is denoted by $\mathbf{x}_{i}$, where $i \in\left\{1, \ldots, N_{V}\right\}$. For the $j$ th PD at the $i$ th VLC unit, 


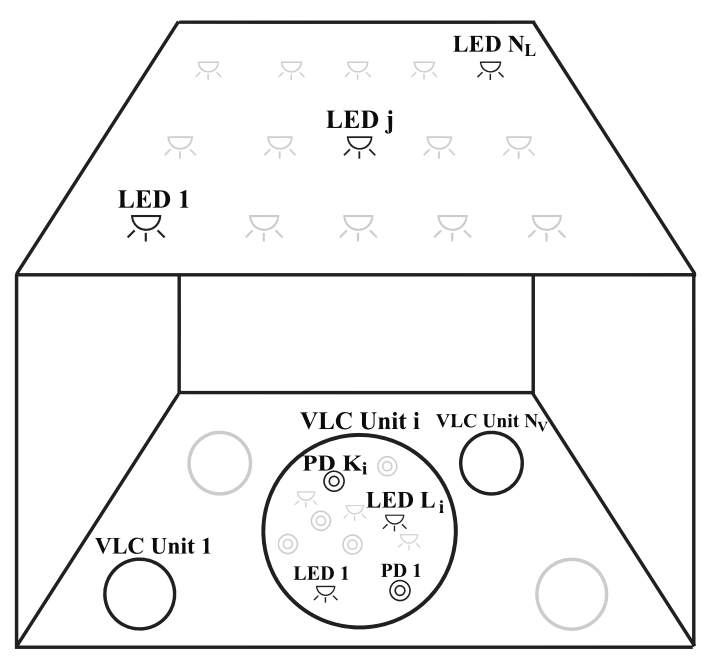

Fig. 1. Cooperative VLP system.

the location is given by $\mathbf{x}_{i}+\mathbf{a}_{i, j}$ and the orientation vector is denoted by $\mathbf{n}_{R, j}^{(i)}$, where $j \in\left\{1, \ldots, K_{i}\right\}$. Similarly, for the $j$ th LED at the $i$ th VLC unit, the location is given by $\mathbf{x}_{i}+\mathbf{b}_{i, j}$ and the orientation vector is represented by $\mathbf{n}_{T, j}^{(i)}$, where $j \in\left\{1, \ldots, L_{i}\right\}$. The displacement vectors, $\mathbf{a}_{i, j}$ 's and $\mathbf{b}_{i, j}$ 's, are known design parameters for the VLC units. Also, the orientation vectors for the LEDs and PDs at the VLC units are assumed to be known since they can be determined by the VLC unit design and by auxiliary sensors (e.g., gyroscope). To distinguish the LED transmitters at known locations from the LEDs at the VLC units, the former are called as the LEDs on the ceiling (as in Fig. 1) in the remainder of the text.

At a given time, each PD can communicate with a subset of all the LEDs in the system. Therefore, the following connectivity sets are defined to specify the connections between the LEDs and the PDs:

$$
\begin{aligned}
& \widetilde{S}_{k}^{(j)}=\left\{l \in\left\{1, \ldots, N_{L}\right\} \mid l\right. \text { th LED on ceiling is } \\
&\text { connected to } k \text { th PD of } j \text { th VLC unit }\} \\
& S_{k}^{(i, j)}=\left\{l \in\left\{1, \ldots, L_{i}\right\} \mid l \text { th LED of } i\right. \text { th VLC unit is } \\
&\text { connected to } k \text { th PD of } j \text { th VLC unit }\} .
\end{aligned}
$$

Namely, $\widetilde{S}_{k}^{(j)}$ represents the set of LEDs on the ceiling that are connected to the $k$ th PD at the $j$ th VLC unit. Similarly, $S_{k}^{(i, j)}$ is the set of LEDs at the $i$ th VLC unit that are connected to the $k$ th PD at the $j$ th VLC unit.

The aim is to estimate the unknown locations, $\mathbf{x}_{1}, \ldots, \mathbf{x}_{N_{V}}$, of the VLC units based on power measurements at the PDs. Let $\widetilde{P}_{l, k}^{(j)}$ represent the power measurement at the $k$ th PD of the $j$ th VLC unit due to the transmission from the $l$ th LED on the ceiling. Similarly, let $P_{l, k}^{(i, j)}$ denote the power measurements at the $k$ th PD of the $j$ th VLC unit due to the $l$ th LED at the $i$ th VLC unit. Based on the Lambertian formula [17], [25], $\widetilde{P}_{l, k}^{(j)}$ and $P_{l, k}^{(i, j)}$ can be expressed as follows:

$$
\widetilde{P}_{l, k}^{(j)}=\frac{\widetilde{m}_{l}+1}{2 \pi} \widetilde{P}_{T, l} \cos ^{\widetilde{m}_{l}}\left(\widetilde{\phi}_{l, k}^{(j)}\right) \cos \left(\widetilde{\theta}_{l, k}^{(j)}\right) \frac{A_{k}^{(j)}}{\left(\widetilde{d}_{l, k}^{(j)}\right)^{2}}+\widetilde{\eta}_{l, k}^{(j)}
$$

for $j \in\left\{1, \ldots, N_{V}\right\}, k \in\left\{1, \ldots, K_{j}\right\}$, and $l \in \widetilde{S}_{k}^{(j)}$, and

$$
\begin{aligned}
P_{l, k}^{(i, j)} & =\frac{m_{l}^{(i)}+1}{2 \pi} P_{T, l}^{(i)} \cos ^{m_{l}^{(i)}}\left(\phi_{l, k}^{(i, j)}\right) \cos \left(\theta_{l, k}^{(i, j)}\right) \frac{A_{k}^{(j)}}{\left(d_{l, k}^{(i, j)}\right)^{2}} \\
& +\eta_{l, k}^{(i, j)}
\end{aligned}
$$

for $j \in\left\{1, \ldots, N_{V}\right\}, k \in\left\{1, \ldots, K_{j}\right\}, i \in\left\{1, \ldots, N_{V}\right\} \backslash j$ and $l \in S_{k}^{(i, j)}$, where the distances $\widetilde{d}_{l, k}^{(j)}$ and $d_{l, k}^{(i, j)}$ are given by

$$
\begin{aligned}
\widetilde{d}_{l, k}^{(j)} & =\left\|\widetilde{\mathbf{d}}_{l, k}^{(j)}\right\| \text { with } \widetilde{\mathbf{d}}_{l, k}^{(j)} \triangleq \mathbf{x}_{j}+\mathbf{a}_{j, k}-\mathbf{y}_{l} \\
d_{l, k}^{(i, j)} & =\left\|\mathbf{d}_{l, k}^{(i, j)}\right\| \text { with } \mathbf{d}_{l, k}^{(i, j)} \triangleq \mathbf{x}_{j}+\mathbf{a}_{j, k}-\mathbf{x}_{i}-\mathbf{b}_{i, l} .
\end{aligned}
$$

In (3) and (4), $\tilde{m}_{l}\left(m_{l}^{(i)}\right)$ is the Lambertian order for the $l$ th LED on the ceiling (at the $i$ th VLC unit), $A_{k}^{(j)}$ is the area of the $k$ th PD at the $j$ th VLC unit, $\widetilde{P}_{T, l}\left(P_{T, l}^{(i)}\right)$ is the transmit power of the $l$ th LED on the ceiling (at the $i$ th VLC unit), $\widetilde{\phi}_{l, k}^{(j)}\left(\phi_{l, k}^{(i, j)}\right)$ is the irradiation angle at the $l$ th LED on the ceiling (at the $i$ th VLC unit) with respect to the $k$ th PD at the $j$ th VLC unit, and $\widetilde{\theta}_{l, k}^{(j)}\left(\theta_{l, k}^{(i, j)}\right)$ is the incidence angle for the $k$ th PD at the $j$ th VLC unit related to the $l$ th LED on the ceiling (at the $i$ th VLC unit). In addition, the noise components, $\widetilde{\eta}_{l, k}^{(j)}$ and $\eta_{l, k}^{(i, j)}$, are modeled by zero-mean Gaussian random variables each with a variance of $\sigma_{j, k}^{2}$. Considering the use of a certain multiplexing scheme (e.g., time division multiplexing among the LEDs at the same VLC unit and on the ceiling, and frequency division multiplexing among the LEDs at different VLC units or on the ceiling), $\widetilde{\eta}_{l, k}^{(j)}$ and $\eta_{l, k}^{(i, j)}$ are assumed to be independent for all different $(j, k)$ pairs and for all $l$ and $i$.

From (5) and (6), the power measurements in (3) and (4) can also be expressed as

$$
\begin{aligned}
\widetilde{P}_{l, k}^{(j)} & =\widetilde{\alpha}_{l, k}^{(j)}\left(\mathbf{x}_{j}\right)+\widetilde{\eta}_{l, k}^{(j)} \\
P_{l, k}^{(i, j)} & =\alpha_{l, k}^{(i, j)}\left(\mathbf{x}_{i}, \mathbf{x}_{j}\right)+\eta_{l, k}^{(i, j)}
\end{aligned}
$$

where

$$
\begin{aligned}
\widetilde{\alpha}_{l, k}^{(j)}\left(\mathbf{x}_{j}\right) \triangleq & \frac{\widetilde{m}_{l}+1}{2 \pi} \widetilde{P}_{T, l} A_{k}^{(j)} \frac{\left(\left(\widetilde{\mathbf{d}}_{l, k}^{(j)}\right)^{T} \widetilde{\mathbf{n}}_{T, l}\right)^{\widetilde{m}_{l}}\left(\widetilde{\mathbf{d}}_{l, k}^{(j)}\right)^{T} \mathbf{n}_{R, k}^{(j)}}{\left\|\widetilde{\mathbf{d}}_{l, k}^{(j)}\right\|^{\widetilde{m}_{l}+3}} \\
\alpha_{l, k}^{(i, j)}\left(\mathbf{x}_{i}, \mathbf{x}_{j}\right) \triangleq & -\frac{m_{l}^{(i)}+1}{2 \pi} P_{T, l}^{(i)} A_{k}^{(j)} \\
& \times \frac{\left(\left(\mathbf{d}_{l, k}^{(i, j)}\right)^{T} \mathbf{n}_{T, l}^{(i)}\right)^{m_{l}^{(i)}}\left(\mathbf{d}_{l, k}^{(i, j)}\right)^{T} \mathbf{n}_{R, k}^{(j)}}{\left\|\mathbf{d}_{l, k}^{(i, j)}\right\|^{m_{l}^{(i)}+3}}
\end{aligned}
$$

Let $\mathbf{x} \triangleq\left[\mathbf{x}_{1}^{T} \ldots \mathbf{x}_{N_{V}}^{T}\right]^{T}$ denote the vector of unknown parameters (which has a size of $3 N_{V} \times 1$ ) and let $\mathbf{P}$ represent a vector consisting of all the measurements in (7) and (8). The elements of $\mathbf{P}$ can be expressed as follows: $\left\{\left\{\left\{\widetilde{P}_{l, k}^{(j)}\right\}_{l \in \widetilde{S}_{k}^{(j)}}\right\}_{k \in\left\{1, \ldots, K_{j}\right\}}\right\}_{j \in\left\{1, \ldots, N_{V}\right\}}$
$\left\{\left\{\left\{\left\{P_{l, k}^{(i, j)}\right\}_{l \in S_{k}^{(i, j)}}\right\}_{i \in\left\{1, \ldots, N_{V}\right\} \backslash\{j\}}\right\}_{k \in\left\{1, \ldots, K_{j}\right\}}\right\}_{j \in\left\{1, \ldots, N_{V}\right\}}$ 
Then, the conditional probability density function (PDF) of $\mathbf{P}$ given $\mathbf{x}$, i.e., the likelihood function, can be stated as

$$
\begin{aligned}
f(\mathbf{P} \mid \mathbf{x}) & =\prod_{j=1}^{N_{V}} \prod_{k=1}^{K_{j}}\left(\prod_{l \in \widetilde{S}_{k}^{(j)}} f\left(\widetilde{P}_{l, k}^{(j)} \mid \mathbf{x}\right)\right. \\
& \left.\times \prod_{i \in\left\{1, \ldots, N_{V}\right\} \backslash\{j\}} \prod_{l \in S_{k}^{(i, j)}} f\left(P_{l, k}^{(i, j)} \mid \mathbf{x}\right)\right)
\end{aligned}
$$

where $f\left(\widetilde{P}_{l, k}^{(j)} \mid \mathbf{x}\right)$ and $f\left(P_{l, k}^{(i, j)} \mid \mathbf{x}\right)$ are the marginal conditional PDFs of $\widetilde{P}_{l, k}^{(j)}$ and $P_{l, k}^{(i, j)}$, respectively. Since $\widetilde{P}_{l, k}^{(j)} \mid \mathbf{x}$ and $P_{l, k}^{(i, j)} \mid \mathbf{x}$ are Gaussian distributed with means of $\widetilde{\alpha}_{l, k}^{(j)}\left(\mathbf{x}_{j}\right)$ and $\alpha_{l, k}^{(i, j)}\left(\mathbf{x}_{i}, \mathbf{x}_{j}\right)$, respectively, and a variance of $\sigma_{j, k}^{2}$ each (see (7) and (8)), (11) can be specified as

$$
\begin{aligned}
& f(\mathbf{P} \mid \mathbf{x})=\prod_{j=1}^{N_{V}} \prod_{k=1}^{K_{j}}\left(\frac{e^{-\frac{1}{2 \sigma_{j, k}^{2}} \sum_{l \in \widetilde{S}_{k}^{(j)}}\left(\widetilde{P}_{l, k}^{(j)}-\widetilde{\alpha}_{l, k}^{(j)}\left(\mathbf{x}_{j}\right)\right)^{2}}}{\left(\sqrt{2 \pi} \sigma_{j, k}\right)^{\left|\widetilde{S}_{k}^{(j)}\right|}}\right) \\
& \left.\times \prod_{i \in\left\{1, \ldots, N_{V}\right\} \backslash\{j\}} \frac{e^{-\frac{1}{2 \sigma_{j, k}^{2}} \sum_{l \in S_{k}^{(i, j)}}\left(P_{l, k}^{(i, j)}-\alpha_{l, k}^{(i, j)}\left(\mathbf{x}_{i}, \mathbf{x}_{j}\right)\right)^{2}}}{\left(\sqrt{2 \pi} \sigma_{j, k}\right)^{\left|S_{k}^{(i, j)}\right|}}\right)
\end{aligned}
$$

where $\left|\widetilde{S}_{k}^{(j)}\right|$ and $\left|S_{k}^{(i, j)}\right|$ represent the number of elements in sets $\widetilde{S}_{k}^{(j)}$ and $S_{k}^{(i, j)}$, respectively. After some manipulation, (12) can be expressed as

$f(\mathbf{P} \mid \mathbf{x})=\left(\prod_{j=1}^{N_{V}} \prod_{k=1}^{K_{j}} \frac{1}{\left(\sqrt{2 \pi} \sigma_{j, k}\right)^{N_{t o t}^{(j, k)}}}\right) e^{-\sum_{j=1}^{N_{V}} \sum_{k=1}^{K_{j}} \frac{h_{j, k}(\mathbf{x})}{2 \sigma_{j, k}^{2}}}$

where $N_{t o t}^{(j, k)}$ represents the total number of LEDs that can communicate with the $k$ th PD at the $j$ th VLC unit; that is,

$$
N_{t o t}^{(j, k)} \triangleq\left|\widetilde{S}_{k}^{(j)}\right|+\sum_{i=1, i \neq j}^{N_{V}}\left|S_{k}^{(i, j)}\right|
$$

and $h_{j, k}(\mathbf{x})$ is defined as

$$
\begin{aligned}
h_{j, k}(\mathbf{x}) \triangleq & \sum_{l \in \widetilde{S}_{k}^{(j)}}\left(\widetilde{P}_{l, k}^{(j)}-\widetilde{\alpha}_{l, k}^{(j)}\left(\mathbf{x}_{j}\right)\right)^{2} \\
& +\sum_{i=1, i \neq j}^{N_{V}} \sum_{l \in S_{k}^{(i, j)}}\left(P_{l, k}^{(i, j)}-\alpha_{l, k}^{(i, j)}\left(\mathbf{x}_{i}, \mathbf{x}_{j}\right)\right)^{2} .
\end{aligned}
$$

From (13), the maximum likelihood estimator (MLE) is obtained as

$$
\hat{\mathbf{x}}_{\mathrm{ML}}=\arg \min _{\mathbf{x}} \sum_{j=1}^{N_{V}} \sum_{k=1}^{K_{j}} \frac{h_{j, k}(\mathbf{x})}{\sigma_{j, k}^{2}}
$$

and the Fisher information matrix (FIM) [26] is given by

$$
[\mathbf{J}]_{t_{1}, t_{2}}=\mathbb{E}\left\{\frac{\partial \log f(\mathbf{P} \mid \mathbf{x})}{\partial x_{t_{1}}} \frac{\partial \log f(\mathbf{P} \mid \mathbf{x})}{\partial x_{t_{2}}}\right\}
$$

where $x_{t_{1}}\left(x_{t_{2}}\right)$ represents element $t_{1}\left(t_{2}\right)$ of vector $\mathbf{x}$ with $t_{1}, t_{2} \in\left\{1,2, \ldots, 3 N_{V}\right\}$. Then, the CRLB is stated as

$$
\operatorname{CRLB}=\operatorname{trace}\left(\mathbf{J}^{-1}\right) \leq \mathbb{E}\left\{\|\hat{\mathbf{x}}-\mathbf{x}\|^{2}\right\}
$$

where $\hat{\mathbf{x}}$ represents an unbiased estimator of $\mathbf{x}$. From (13) and (15), the elements of the FIM in (17) can be calculated after some manipulation as

$$
\begin{aligned}
{[\mathbf{J}]_{t_{1}, t_{2}} } & =\sum_{j=1}^{N_{V}} \sum_{k=1}^{K_{j}} \frac{1}{\sigma_{j, k}^{2}}\left(\sum_{l \in \widetilde{S}_{k}^{(j)}} \frac{\partial \widetilde{\alpha}_{l, k}^{(j)}\left(\mathbf{x}_{j}\right)}{\partial x_{t_{1}}} \frac{\partial \widetilde{\alpha}_{l, k}^{(j)}\left(\mathbf{x}_{j}\right)}{\partial x_{t_{2}}}\right. \\
& \left.+\sum_{i=1, i \neq j}^{N_{V}} \sum_{l \in S_{k}^{(i, j)}} \frac{\partial \alpha_{l, k}^{(i, j)}\left(\mathbf{x}_{i}, \mathbf{x}_{j}\right)}{\partial x_{t_{1}}} \frac{\partial \alpha_{l, k}^{(i, j)}\left(\mathbf{x}_{i}, \mathbf{x}_{j}\right)}{\partial x_{t_{2}}}\right) .
\end{aligned}
$$

In addition, from (5), (6), (9), and (10), the partial derivatives in (19) are obtained as follows:

$$
\begin{aligned}
& \frac{\partial \widetilde{\alpha}_{l, k}^{(j)}\left(\mathbf{x}_{j}\right)}{\partial x_{t}}=-\frac{\left(\widetilde{m}_{l}+1\right) \widetilde{P}_{T, l} A_{k}^{(j)}\left(\left(\widetilde{\mathbf{d}}_{l, k}^{(j)}\right)^{T} \widetilde{\mathbf{n}}_{T, l}\right)^{\widetilde{m}_{l}}}{2 \pi\left\|\widetilde{\mathbf{d}}_{l, k}^{(j)}\right\|^{\widetilde{m}_{l}+3}} \\
& \quad \times\left(\widetilde{m}_{l} \widetilde{n}_{T, l}(t-3 j+3)\left(\widetilde{\mathbf{d}}_{l, k}^{(j)}\right)^{T} \mathbf{n}_{R, k}^{(j)}\left(\left(\widetilde{\mathbf{d}}_{l, k}^{(j)}\right)^{T} \widetilde{\mathbf{n}}_{T, l}\right)^{-1}\right. \\
& \quad+n_{R, k}^{(j)}(t-3 j+3) \\
& \left.\quad-\left(\widetilde{m}_{l}+3\right) \widetilde{d}_{l, k}^{(j)}(t-3 j+3)\left(\widetilde{\mathbf{d}}_{l, k}^{(j)}\right)^{T} \mathbf{n}_{R, k}^{(j)}\left\|\widetilde{\mathbf{d}}_{l, k}^{(j)}\right\|^{-2}\right)
\end{aligned}
$$

for $t \in\{3 j-2,3 j-1,3 j\}$ and $\partial \widetilde{\alpha}_{l, k}^{(j)}\left(\mathbf{x}_{j}\right) / \partial x_{t}=0$ otherwise, where $\widetilde{n}_{T, l}(t-3 j+3), n_{R, k}^{(j)}(t-3 j+3)$, and $\widetilde{d}_{l, k}^{(j)}(t-3 j+3)$ represent the $(t-3 j+3)$ th elements of $\widetilde{\mathbf{n}}_{T, l}, \mathbf{n}_{R, k}^{(j)}$, and $\widetilde{\mathbf{d}}_{l, k}^{(j)}$, respectively. Similarly,

$$
\begin{aligned}
& \frac{\partial \alpha_{l, k}^{(i, j)}\left(\mathbf{x}_{i}, \mathbf{x}_{j}\right)}{\partial x_{t}}=-\frac{\left(m_{l}^{(i)}+1\right) P_{T, l}^{(i)} A_{k}^{(j)}\left(\left(\mathbf{d}_{l, k}^{(i, j)}\right)^{T} \mathbf{n}_{T, l}^{(i)}\right)^{m_{l}^{(i)}}}{2 \pi\left\|\mathbf{d}_{l, k}^{(i, j)}\right\|_{l}^{m_{l}^{(i)}+3}} \\
& \times\left(m_{l}^{(i)} n_{T, l}^{(i)}(t-3 j+3)\left(\mathbf{d}_{l, k}^{(i, j)}\right)^{T} \mathbf{n}_{R, k}^{(j)}\left(\left(\mathbf{d}_{l, k}^{(i, j)}\right)^{T} \mathbf{n}_{T, l}^{(i)}\right)^{-1}\right. \\
& \quad+n_{R, k}^{(j)}(t-3 j+3) \\
& \left.\quad-\left(m_{l}^{(i)}+3\right) d_{l, k}^{(i, j)}(t-3 j+3)\left(\mathbf{d}_{l, k}^{(i, j)}\right)^{T} \mathbf{n}_{R, k}^{(j)}\left\|\mathbf{d}_{l, k}^{(i, j)}\right\|^{-2}\right)
\end{aligned}
$$

for $t \in\{3 j-2,3 j-1,3 j\}, \partial \alpha_{l, k}^{(i, j)}\left(\mathbf{x}_{i}, \mathbf{x}_{j}\right) / \partial x_{t}$ is equal to the negative of (21) with $(t-3 j+3)$ 's being replaced by $(t-3 i+$ $3)$ 's for $t \in\{3 i-2,3 i-1,3 i\}$, and $\partial \alpha_{l, k}^{(i, j)}\left(\mathbf{x}_{i}, \mathbf{x}_{j}\right) / \partial x_{t}=0$ otherwise. In (21), $n_{T, l}^{(i)}(t-3 j+3)$ and $d_{l, k}^{(i, j)}(t-3 j+3)$ denote the $(t-3 j+3)$ th elements of $\mathbf{n}_{T, l}^{(i)}$ and $\mathbf{d}_{l, k}^{(i, j)}$, respectively.

Based on (18)-(21), the CRLB for location estimation can be obtained for cooperative VLP systems. The obtained CRLB expression is generic for any three-dimensional configuration and covers all possible cooperation scenarios via the definitions of the connectivity sets (see (1) and (2)). To the best of authors' knowledge, no such CRLB expressions have been available in the literature for cooperative VLP systems.

Remark 1: From (19), it is noted that the first summation term in the parentheses is related to the information from the LED transmitters on the ceiling whereas the remaining terms are due to the cooperation among the VLC units. In the noncooperative case, the elements of the FIM are given by the expression in the first line of (19).

Via (18)-(21), the effects of cooperation on the accuracy of VLP systems can be quantified, as investigated next. 


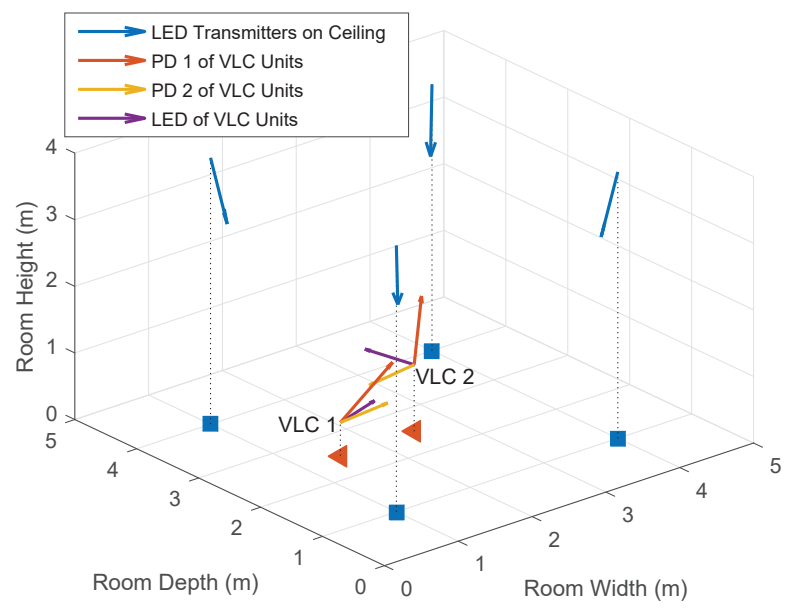

Fig. 2. VLP network configuration in the simulations. Each VLC unit contains two PDs and one LED. PD 1 of the VLC units is used to obtain measurements from the LEDs on the ceiling while PD 2 of the VLC units communicates with the LED of the other VLC unit for cooperative localization. The squares and the triangles show the projections of the LEDs and the VLC units on the floor, respectively.

\section{NUMERICAL RESULTS}

In this section, theoretical bounds on cooperative localization are investigated to illustrate the effects of cooperation on localization accuracy. VLP system parameters are determined in a similar way to the studies in [17] and [19]. The area of each PD is set to $1 \mathrm{~cm}^{2}$ and the Lambertian order of all the LEDs is selected as $m=1$. In addition, the noise variances are calculated using [27, Eq. 6] and [28, Eq. 20]. The parameters for noise variance calculation are set to be the same as those used in [27] (see Table I in [27]).

The VLP system considered in the simulations is illustrated in Fig. 2. A room of size $5 \mathrm{~m} \times 5 \mathrm{~m} \times 4 \mathrm{~m}$ is considered, where there exist $N_{L}=4$ LED transmitters on the ceiling which are located at $\mathbf{y}_{1}=\left[\begin{array}{lll}1 & 1 & 4\end{array}\right]^{T} \mathrm{~m} ., \mathbf{y}_{2}=\left[\begin{array}{lll}1 & 4 & 4\end{array}\right]^{T} \mathrm{~m}$., $\mathbf{y}_{3}=\left[\begin{array}{lll}4 & 1 & 4\end{array}\right]^{T} \mathrm{~m}$. , and $\mathbf{y}_{4}=\left[\begin{array}{lll}4 & 4 & 4\end{array}\right]^{T} \mathrm{~m}$. The orientations of the LEDs on the ceiling are adjusted so that each LED is directed towards the center of the room with an offset angle of $10^{\circ}$ with respect to the normal vector of the ceiling. In addition to the LEDs on the ceiling, there exist $N_{V}=2$ VLC units whose locations are given by $\mathbf{x}_{1}=\left[\begin{array}{llll}2.5 & 2.5 & 1\end{array}\right]^{T} \mathrm{~m}$. and $\mathbf{x}_{2}=\left[\begin{array}{lll}1.5 & 2.5 & 0.5\end{array}\right]^{T} \mathrm{~m}$. Each VLC unit consists of two PDs and one LED, with offsets with respect to the center of the VLC unit being set to $\mathbf{a}_{j, 1}=\left[\begin{array}{lll}0 & -0.1 & 0\end{array}\right]^{T} \mathrm{~m} ., \mathbf{a}_{j, 2}=\left[\begin{array}{lll}0 & 0.1 & 0\end{array}\right]^{T} \mathrm{~m}$., and $\mathbf{b}_{j, 1}=\left[\begin{array}{lll}0.1 & 0 & 0\end{array}\right]^{T} \mathrm{~m}$. for $j=1,2$. The orientation vectors of the PDs and the LEDs on the VLC units are obtained as the normalized versions (the orientation vectors are unit-norm) of the following vectors: $\mathbf{n}_{R, 1}^{(1)}=\left[\begin{array}{lll}0.1 & 0 & 1\end{array}\right]^{T}$, $\mathbf{n}_{R, 1}^{(2)}=\left[\begin{array}{lll}0.6 & -0.31\end{array}\right]^{T}, \mathbf{n}_{R, 2}^{(1)}=\left[\begin{array}{lll}-0.9 & 0.2 & -0.3\end{array}\right]^{T}, \mathbf{n}_{R, 2}^{(2)}=$ $\left[\begin{array}{lll}0.2 & -0.1 & 0.1\end{array}\right]^{T}, \mathbf{n}_{T, 1}^{(1)}=\left[\begin{array}{lll}-0.2 & 0.3 & 0.1\end{array}\right]^{T}$, and $\mathbf{n}_{T, 1}^{(2)}=$ $\left[\begin{array}{lll}0.6 & 0.1 & 0.1\end{array}\right]^{T}$. Furthermore, the connectivity sets are defined as $S_{1}^{(i, j)}=\emptyset, S_{2}^{(i, j)}=\{1\}$ for $i, j \in\{1,2\}, i \neq j$ in the cooperative case and $\widetilde{S}_{1}^{(j)}=\{1,2,3,4\}, \widetilde{S}_{2}^{(j)}=\emptyset$ for $j \in\{1,2\}$ in the noncooperative case.

In order to analyze the localization performance of the VLC units with respect to the transmit powers of the LEDs on the ceiling (equivalently, anchors), individual CRLBs for localization of the VLC units in noncooperative and cooperative scenarios are plotted against the transmit powers of LEDs on ceiling in Fig. 3, where the transmit power of the VLC units is fixed to $1 \mathrm{~W}$. As observed from Fig. 3, the CRLBs in the cooperative scenario converge to those in the noncooperative scenario as the transmit powers of the LEDs increase. Since the first (second) summand in the FIM expression in (19) corresponds to the noncooperative (cooperative) localization, higher transmit powers of the LEDs on the ceiling cause the first summand to be much greater than the second summand, which makes the contribution of cooperation to the FIM negligible. Hence, the effect of cooperation on localization performance becomes less significant as the transmit power increases, which is in compliance with the results obtained for RF based cooperative localization networks [7]. In addition, it is observed from Fig. 3 that the improvement in localization accuracy gained by employing cooperation among the VLC units is higher for VLC 2 as compared to that for VLC 1 . This is an intuitive result since the localization of VLC 2 depends mostly on LED 2 (the other LEDs are not sufficiently close to facilitate the localization process), and incorporating cooperative measurements for VLC 2 provides an improvement in localization performance that is much greater than that for VLC 1, which can obtain informative measurements from the LEDs on the ceiling even in the absence of cooperation.

Finally, the localization performance of the VLC units is investigated with respect to the transmit powers of the VLC units when the transmit powers of the LEDs on the ceiling are fixed. Fig. 4 illustrates the CRLBs for localization of the VLC units versus the transmit powers of the VLC units in the noncooperative and cooperative cases. As observed from Fig. 4, cooperation leads to a higher improvement in the performance of VLC 2, similar to Fig. 3. In addition, via the FIM expression in (19), it can be noted that the contribution of cooperation to localization performance gets higher as the transmit powers of the VLC units increase, which is also observed from Fig. 4. However, the CRLB reaches a saturation level above a certain power threshold, as opposed to Fig. 3, where the CRLB continues to decrease as the power increases. The main reason for this distinction between the effects of the transmit powers of the LEDs on the ceiling and those of the VLC units can be explained as follows: For a fixed transmit power of the VLC units, the localization error in a threedimensional scenario by using four anchors (i.e., four LEDs on the ceiling) converges to zero as the transmit powers of the anchors increase regardless of the existence of cooperation. On the other hand, for a fixed transmit power of the LEDs on the ceiling, increasing the transmit power of the VLC unit (i.e., one of the anchors) cannot reduce the localization error below a certain level. Therefore, the saturation level represents the localization accuracy that can be attained by five anchors with four anchors leading to noisy RSS measurements and one anchor generating noise-free RSS measurements.

\section{CONCLUDING REMARKS}

A cooperative VLP system has been proposed based on a generic system model consisting of LED transmitters at known locations and VLC units with multiple LEDs and PDs. The 


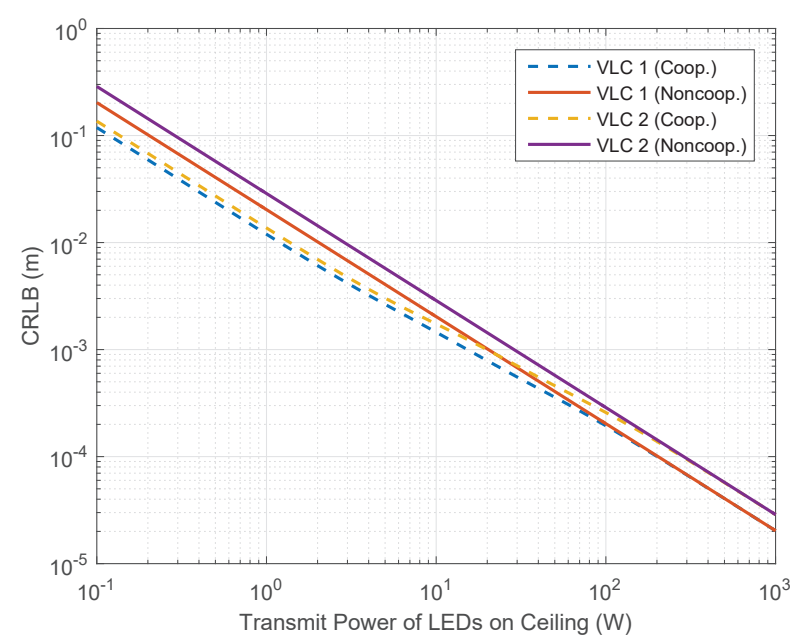

Fig. 3. Individual CRLBs for localization of VLC units in both noncooperative and cooperative cases with respect to the transmit power of LEDs on ceiling, where the transmit power of VLC units is taken as $1 \mathrm{~W}$.

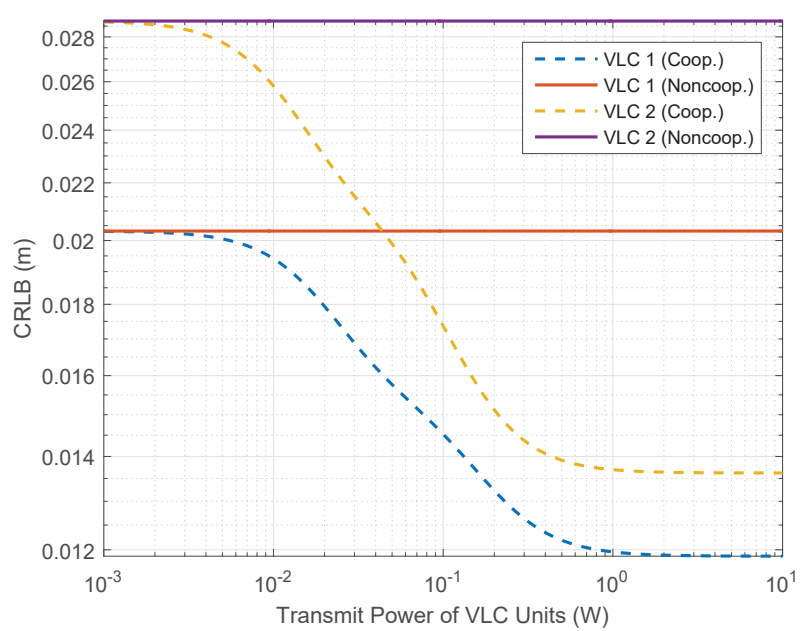

Fig. 4. Individual CRLBs for localization of VLC units in both noncooperative and cooperative cases with respect to the transmit power of VLC units, where the transmit power of LEDs on ceiling is taken as $1 \mathrm{~W}$.

effects of cooperation on the localization accuracy of a VLP systems have been quantified based on a CRLB expression, which is generic for any three-dimensional configuration and covers all possible cooperation scenarios via definitions of connectivity sets. Numerical results have been presented and significance of cooperation in various scenarios has been investigated.

\section{REFERENCES}

[1] G. Mao and B. Fidan, Localization Algorithms and Strategies for Wireless Sensor Networks. Information Science Reference, 2009.

[2] K. Pahlavan and P. Krishnamurthy, Principles of Wireless Access and Localization, ser. Wiley Desktop Editions. Wiley, 2013.

[3] H. Liu, H. Darabi, P. Banerjee, and J. Liu, "Survey of wireless indoor positioning techniques and systems," IEEE Transactions on Systems, Man, and Cybernetics, Part C (Applications and Reviews), vol. 37, no. 6, pp. 1067-1080, Nov. 2007.

[4] M. Cypriani, F. Lassabe, P. Canalda, and F. Spies, "Wi-Fi-based indoor positioning: Basic techniques, hybrid algorithms and open software platform," in International Conference on Indoor Positioning and Indoor Navigation (IPIN), Sep. 2010.
[5] Z. Sahinoglu, S. Gezici, and I. Guvenc, Ultra-wideband Positioning Systems: Theoretical Limits, Ranging Algorithms, and Protocols. New York: Cambridge University Press, 2008.

[6] J. Armstrong, Y. Sekercioglu, and A. Neild, "Visible light positioning: A roadmap for international standardization," IEEE Communications Magazine, vol. 51, no. 12, pp. 68-73, Dec. 2013.

[7] M. R. Gholami, M. F. Keskin, S. Gezici, and M. Jansson, Cooperative Positioning in Wireless Networks. Wiley Encyclopedia of Electrical and Electronics Engineering, 2016, pp. 1-19.

[8] R. M. Vaghefi, M. R. Gholami, R. M. Buehrer, and E. G. Strom "Cooperative received signal strength-based sensor localization with unknown transmit powers," IEEE Transactions on Signal Processing, vol. 61, no. 6, pp. 1389-1403, Mar. 2013.

[9] A. Conti, M. Guerra, D. Dardari, N. Decarli, and M. Z. Win, "Network experimentation for cooperative localization," IEEE Journal on Selected Areas in Communications, vol. 30, no. 2, pp. 467-475, Feb. 2012

[10] Y. Shen, H. Wymeersch, and M. Z. Win, "Fundamental limits of wideband cooperative localization via Fisher information," in IEEE Wireless Commun. and Networking Conf., Mar. 2007, pp. 3951-3955.

[11] C. L. F. Mayorga, F. della Rosa, S. A. Wardana, G. Simone, M. C. N. Raynal, J. Figueiras, and S. Frattasi, "Cooperative positioning techniques for mobile localization in 4G cellular networks," in IEEE International Conference on Pervasive Services, July 2007, pp. 39-44.

[12] E. D. Nerurkar, S. I. Roumeliotis, and A. Martinelli, "Distributed maximum a posteriori estimation for multi-robot cooperative localization," in IEEE International Conference on Robotics and Automation, May 2009, pp. 1402-1409.

[13] R. M. Vaghefi and R. M. Buehrer, "Joint TOA-based sensor synchronization and localization using semidefinite programming," in IEEE Int. Conference on Commun. (ICC), June 2014, pp. 520-525.

[14] M. R. Gholami, S. Gezici, and E. G. Strom, "Improved position estimation using hybrid TW-TOA and TDOA in cooperative networks," IEEE Transactions on Signal Processing, vol. 60, no. 7, pp. 3770-3785, July 2012.

[15] H. Wymeersch, J. Lien, and M. Z. Win, "Cooperative localization in wireless networks," Proceedings of the IEEE, vol. 97, no. 2, pp. 427 450, Feb. 2009.

[16] M. R. Gholami, L. Tetruashvili, E. G. Strm, and Y. Censor, "Cooperative wireless sensor network positioning via implicit convex feasibility," IEEE Transactions on Signal Processing, vol. 61, no. 23, pp. 58305840, Dec. 2013.

[17] T. Wang, Y. Sekercioglu, A. Neild, and J. Armstrong, "Position accuracy of time-of-arrival based ranging using visible light with application in indoor localization systems," Journal of Lightwave Technology, vol. 31, no. 20, pp. 3302-3308, Oct. 2013.

[18] X. Zhang, J. Duan, Y. Fu, and A. Shi, "Theoretical accuracy analysis of indoor visible light communication positioning system based on received signal strength indicator," Journal of Lightwave Technology, vol. 32 no. 21 , pp. $4180-4186$, Nov. 2014

[19] M. F. Keskin and S. Gezici, "Comparative theoretical analysis of distance estimation in visible light positioning systems," Journal of Lightwave Technology, vol. 34, no. 3, pp. 854-865, Feb. 2016.

[20] M. F. Keskin, E. Gonendik, and S. Gezici, "Improved lower bounds for ranging in synchronous visible light positioning systems," Journal of Lightwave Technology, vol. 34, no. 23, pp. 5496-5504, Dec. 2016.

[21] E. Gonendik and S. Gezici, "Fundamental limits on RSS based range estimation in visible light positioning systems," IEEE Communications Letters, vol. 19, no. 12, pp. 2138-2141, Dec. 2015.

[22] A. Sahin, Y. S. Eroglu, I. Guvenc, N. Pala, and M. Yuksel, "Hybrid 3-D localization for visible light communication systems," Journal of Lightwave Technology, vol. 33, no. 22, pp. 4589-4599, Nov. 2015.

[23] H. Steendam, T. Q. Wang, and J. Armstrong, "Theoretical lower bound for indoor visible light positioning using received signal strength measurements and an aperture-based receiver," Journal of Lightwave Technology, vol. 35, no. 2, pp. 309-319, Jan. 2017.

[24] M. Yasir, S. W. Ho, and B. N. Vellambi, "Indoor positioning system using visible light and accelerometer," Journal of Lightwave Technology, vol. 32 , no. 19 , pp. 3306-3316, Oct. 2014

[25] L. Li, P. Hu, C. Peng, G. Shen, and F. Zhao, "Epsilon: A visible light based positioning system," in 11th USENIX Symposium on Networked Systems Design and Implementation, Seattle, WA, 2014, pp. 331-343.

[26] H. V. Poor, An Introduction to Signal Detection and Estimation. New York: Springer-Verlag, 1994.

[27] H. Ma, L. Lampe, and S. Hranilovic, "Coordinated broadcasting for multiuser indoor visible light communication systems," IEEE Transactions on Communications, vol. 63, no. 9, pp. 3313-3324, Sep. 2015.

[28] T. Komine and M. Nakagawa, "Fundamental analysis for visible-light communication system using LED lights," IEEE Transactions on Consumer Electronics, vol. 50, no. 1, pp. 100-107, Feb. 2004. 